\title{
Family History and Stroke Risk in China: Evidence from a Large Cohort Study
}

\author{
Tian Tian, ${ }^{\mathrm{a}}$ Guangfu Jin, ${ }^{\mathrm{a}}$ Canqing Yu, ${ }^{\mathrm{b}}$ Jun Lv, ${ }^{\mathrm{b}}$ Yu Guo, ${ }^{\mathrm{c}}$ Zheng Bian, ${ }^{\mathrm{c}}$ Ling Yang, ${ }^{\mathrm{d}}$ Yiping Chen, ${ }^{\mathrm{d}}$ \\ Hongbing Shen, ${ }^{a}$ Zhengming Chen, ${ }^{a}$ Zhibin $\mathrm{Hu}^{\mathrm{a}}{ }^{\mathrm{a}}$ Liming $\mathrm{Li}^{\mathrm{b}, \mathrm{c}}$ on behalf of the China Kadoorie Biobank \\ Collaborative Group \\ a'Department of Epidemiology and Biostatistics, School of Public Health, Nanjing Medical University, Nanjing, China \\ ${ }^{b}$ Department of Epidemiology and Biostatistics, School of Public Health, Peking University Health Science Center, Beijing, China \\ 'Chinese Academy of Medical Sciences, Beijing, China \\ ${ }^{d}$ Clinical Trial Service Unit \& Epidemiological Studies Unit (CTSU), Nuffield Department of Population Health, University of Oxford, Oxford, UK
}

Background and Purpose Large cohort studies on relationship between family history of stroke (FHS) and stroke risk are lacking in Asians. We aimed to systematically evaluate the association of FHS with stroke risk in a cohort study of 0.5 million Chinese adults.

Methods Information about FHS was self-reported. The median follow-up time was 7.16 years and the end-point of follow-up was incident stroke, which was entered directly into the China Kadoorie Biobank system. Multivariate analyses were performed with Cox proportional hazards model, and interaction analyses were carried using likelihood-ratio tests.

Results Compared with participants without FHS, the hazard ratio (HR) (95\% confidence interval, $\mathrm{Cl}$ ) of stroke for participants with FHS was 1.50 (1.46-1.55). The HRs increased with the number of first degree relatives with stroke (HRs=1.41, 1.98 and 2.47 for 1, 2 and $\geq 3$ relatives, respectively, $\left.P_{\text {trend }}<0.001\right)$. The HRs were 1.57 (95\% Cl: $\left.1.50-1.66\right)$ and 1.49 (95\% Cl: $\left.1.45-1.54\right)$ for sibling history and parental history, respectively. Similar associations with offspring stroke risk were observed between paternal history ( $\mathrm{HR}=1.48,95 \% \mathrm{Cl}$ : 1.43-1.54) and maternal history ( $\mathrm{HR}=1.49$, 95\% Cl: 1.43-1.55). Moreover, significant interactions were detected between FHS and health-risk behaviors (tobacco smoking and alcohol drinking).

Conclusions FHS is an independent risk factor for stroke in Chinese. The more first degree relatives are affected by stroke, the higher are individuals' risk of suffering from stroke. The management of the health-risk behaviors for reducing stroke should be highlighted, especially for the individuals with FHS.

Keywords Family history; Stroke; Association; Cohort study
Correspondence: Zhibin Hu Department of Epidemiology and Biostatistics, School of Public Health, Nanjing Medical University, 101 Longmian Avenue, Nanjing 211166, China

Tel: +86-25-86868440

Fax: +86-25-86868440

E-mail: zhibin_hu@njmu.edu.cn

Liming Li

Department of Epidemiology and Biostatistics, School of Public Health, Peking University Health

Science Center, 38 Xueyuan Road, Beijing 100191, China

Tel: $+86-10-82801528$

Fax: +86-10-82801528

E-mail: Imlee@vip.163.com

Received: September 3, 2016 Revised: January 28, 2017 Accepted: April 5, 2017

This work was supported by grant from the National Natural Science Foundation of China $(81390540,81390543)$. The CKB baseline survey and the first re-survey were supported by a grant from the Kadoorie Charitable Foundation in Hong Kong. The long-term follow-up is supported by grants from the UK Wellcome Trust (088158/Z/09/Z and $104085 / Z / 14 / Z$ ) and a grant from the Chinese Ministry of Science and Technology (2011BAl09B01). The funders had no role in the study design, data collection, data analysis and interpretation, writing of the report, or the decision to submit the article for publication.

The authors have no financial conflicts of interest. 


\section{Introduction}

Stroke is the second most frequent cause of death worldwide. ${ }^{1}$ In China, there is 2.5 million new stroke cases annually and 7.5 million stroke survivors. ${ }^{2}$ Therefore, it is important to explore more efficient and targeted prevention measures to reduce the stroke incidence.

One convenient way to determine the individuals with potential risks for stroke is to collect family history information. Regularly updating family histories was recommended by the 2002 American Heart Association guidelines for primary prevention of cardiovascular disease. ${ }^{3}$ A series of cohort studies have consistently showed the association of family history of stroke (FHS) with increased risk of stroke, but the effects of FHS varied with the different studies. ${ }^{4-13}$ This may partly be due to the population discrepancy and small sample size (the largest one including 53,691 participants ${ }^{4}$ ). To date, only one Chinese cohort study with 15,131 participants and one Japanese cohort study with 53,691 individuals focused on this association in Asians. ${ }^{4,13}$

The differences have been reported between different types of FHS (maternal, paternal, and sibling history) in relationship with incident stroke. ${ }^{7,14-18}$ Sibling history was more strongly correlated with incident stroke than parental history, ${ }^{14}$ although the difference was not always observed..$^{15}$ The risk of incident stroke was also different in individuals with maternal history versus paternal history. ${ }^{716-19}$ However, the cohort study is still rare to investigate the contributions of different types of FHS to stroke, especially in Chinese population.

Therefore, we intend to systematically evaluate the relationship between FHS and incident stroke in a large-scale, Chinese population-based cohort study.

\section{Methods}

\section{Study population}

This study was based on the China Kadoorie Biobank (CKB, known previously as the Kadoorie Study of Chronic Disease in China [KSCDC]). The study rationale, design, survey methods, and baseline population characteristics of the CKB study have been previously described in detail. ${ }^{20,21}$ In brief, 512,891 participants aged 30-79 years were enrolled between 2004 and 2008 in 10 regions of China. In this study, we excluded 2,577 persons with cancer, 15,472 persons with heart disease, and 8,884 persons with stroke at baseline. In addition, 14,543 individuals with no information about FHS (maternal, paternal or sibling history of stroke) at baseline were also excluded. The final analyses included 473,849 individuals (192,834 men and
281,015 women).

Ethical approval for our study was obtained from Central Ethical Committee of the Chinese Center for Disease Control and Prevention (Beijing, China), and the Oxford Tropical Research Ethics Committee, the University of Oxford (UK). All participants provided written informed consent.

\section{Ascertainment of outcomes}

The end point during the follow-up was incident stroke, including mortality and morbidity data. The vital status of study participants was ascertained through linkage with local disease surveillance points system death certificates and official residential records. Any deaths occurred were coded by trained staff "blinded" to baseline information. Causes of death provided by official death certificates were supplemented, if necessary, separate active confirmation could be carried out by reviewing medical records, reviewing residential records, visiting local communities, or directly contacting participants. Besides, linkage to a local health insurance database was also an important supplementary way of ascertaining deaths. For any additional deaths not verified by routine procedures, the causes could be identified through reviewing hospital records or conducting a verbal autopsy. Information on non-fatal stroke was collected by means of linkage with the established disease registries, as well as the national health insurance claim databases. In addition, participants who failed to be included in the health insurance system were followed annually by trained staff to ascertain their status including hospital admission, disease development, migration and death.

Incident strokes were coded as ischemic stroke (163), hemorrhagic stroke (subarachnoid [160] or intracerebral [161]) and other or unknown stroke type (164) by trained staff "blinded" to baseline information (using the 10th International Classification of Diseases, ICD-10). Besides, stroke cases were further reviewed by a group of professional neurologists according to uniform and standardized diagnostic criteria. Follow-up time was calculated for each participant from the date of the start of study until the date of the occurrence of stroke, loss to follow-up, or the end of follow-up (December 31, 2013), whichever occurred first.

\section{Assessment of FHS}

In the baseline questionnaire, each subject was asked by the interviewer whether the family member (father, mother, or siblings) had been affected by stroke. For siblings, the number of affected members with stroke was recorded. We defined a participant as "family history positive" if he or she reported that one parent or sibling had stroke. Positive parental history was 
defined as reporting a positive history for either or both parents. In this study, first degree relatives included fathers, mothers, and siblings. For each subject, the first degree family members' stroke history had been collected.

\section{Assessment of covariates}

All participants completed the standardized questionnaires covering detailed questions on general socioeconomic and demographic status, health status and medical history (hypertension, diabetes, stroke or transient ischemic attack, cancer and heart disease), smoking status, alcohol consumption, and other lifestyle behaviors. Variables involving general socioeconomic and demographic status included age, sex, residential area, occupation, etc. The level of physical activity was calculated as metabolic equivalent task hours daily (metabolic equivalent of task-hours/day). Besides, the number of siblings was covered.

Physical measurements were made by trained staff using calibrated instruments including body height, weight, waist and hip circumference, heart rate and blood pressure. Body mass index was calculated as weight $(\mathrm{kg})$ divided by the square of height $\left(\mathrm{m}^{2}\right)$. Prevalent diabetes was defined as measured fasting blood glucose $37.0 \mathrm{mmol} / \mathrm{L}$, measured random blood glucose $311.1 \mathrm{mmol} / \mathrm{L}$, or self-reported diagnosis of diabetes. Prevalent hypertension was defined as measured systolic blood pressure $3,140 \mathrm{~mm} \mathrm{Hg}$, measured diastolic blood pressure 390 $\mathrm{mm} \mathrm{Hg}$, self-reported diagnosis of hypertension, or self-reported use of antihypertensive agents at baseline.

\section{Statistical analyses}

Continuous data were presented as mean (standard deviation) and categorical variables as counts and frequencies. We compared means by the method of Student's t-test, and categorical variables were compared using Pearson's $\chi^{2}$ statistic to determine differences in the baseline characteristics between subjects with and without FHS. The Cox proportional hazards regression model was used to evaluate hazard ratios (HRs) and 95\% confidence intervals ( $\mathrm{Cls}$ ) of stroke for FHS. We also estimated the HRs of stroke according to the status of FHS (without FHS, with FHS, with 1 , with 2 , or with $\geq 3$ ill family members). Besides, the HRs were evaluated according to types of FHS (including father, mother, and sibling history). Analyses were done for all incident stroke and also separately for each stroke type. The multivariate adjusted HRs of stroke were estimated with the following adjustments: age (continuous); sex (male or female); residential area (rural or urban); level of education (no formal school, primary school, middle school, high school, technical school/college, or university); marital status (married, widowed, divorced/separated, or never married); alco- hol consumption (non-drinker, occasional drinker, ex-drinker, or current regular drinker); smoking status (never smoker, occasional smoker, ex-smoker, or current regular smoker); physical activity (continuous); body mass index (BMI) (continuous); prevalent hypertension and diabetes at baseline (presence or absence) and number of siblings (continuous).

Stratified analyses were carried on: age $(<45,45$ to 54,55 to 64 , or $365 \mathrm{yr}$ ), gender (male or female), marital status (married or widowed/separated/divorced/never married), residential area (rural or urban), education (Illiteracy/primary school or middle school and above), smoking status (current regular smoker or not), alcohol consumption (current regular drinker or not), physical activity (categorized using median), BMI (<24.0, 24.0 to 27.9 , or 328.0 ), and prevalent hypertension and diabetes at baseline (presence or absence). The interaction analyses were performed by means of likelihood-ratio tests, comparing models with and without the interaction items between the baseline stratifying variables and FHS as a dichotomous variable.

All Cls were estimated at the 95\% level. All statistical tests were two-sided and significance was defined as $P<0.05$. The statistical analyses were performed with $\mathrm{R}$ software (version 3.1.1, 2014-07-10; R Foundation for Statistical Computing, http://www.cran.r-project.org/).

\section{Results}

Baseline characteristics of the study population were summarized in Table 1. The proportion of participants with FHS was $18.15 \%$. Compared with participants without FHS, those with FHS were more likely to be men, older, in urban areas, currently married, highly educated, drink and smoke, and more likely to have a higher BMI, lower physical activity, and hypertension or diabetes problem.

Total person-years of follow-up were 3,338,261 and the median follow-up time was 7.16 years. A total of 26,395 (5.57\%) stroke cases were reported $(20,528$ of ischemic stroke, 4,968 of hemorrhagic stroke and 899 of other conditions). Incidence rate of stroke per 1,000 person years was 7.31 for participants without FHS, and 10.62 for participants with FHS. Incidence rates of stroke according to the number of family members with stroke were $9.78,15.44$, and 24.29 per 1,000 person years for subjects whose FHS involving 1, 2, and $\geq 3$ members, respectively (Table 2). The trend that the incidence of stroke increased with the number of the first degree relatives with stroke was similar for both hemorrhagic and ischemic stroke.

The age- and gender- adjusted HR $(95 \% \mathrm{Cl})$ of stroke for participants with FHS was 1.50 (1.46-1.55) as compared with those without FHS (Table 2). According to the number of family 
Table 1. Baseline characteristics of the study population according to family history of stroke

\begin{tabular}{|c|c|c|c|c|}
\hline \multirow{2}{*}{ Characteristic } & \multirow{2}{*}{ All subjects } & \multicolumn{2}{|c|}{ Family history of stroke } & \multirow{2}{*}{$P^{*}$} \\
\hline & & No & Yes & \\
\hline No. of participants & $473,849(100.0)$ & $387,864(81.9)$ & $85,985(18.1)$ & \\
\hline Age (yr) & $50.8 \pm 10.5$ & $50.8 \pm 10.7$ & $51.1 \pm 9.7$ & $<0.001$ \\
\hline Male & $192,834(40.7)$ & $157,379(40.6)$ & $35,455(41.2)$ & $<0.001$ \\
\hline Rural area & $269,040(56.8)$ & $223,874(57.7)$ & $45,166(52.5)$ & $<0.001$ \\
\hline Currently married & $431,697(91.1)$ & $353,006(91.0)$ & $78,691(91.5)$ & $<0.001$ \\
\hline Middle school and above & $234,959(49.6)$ & $186,132(48.0)$ & 48,827 (56.8) & $<0.001$ \\
\hline Body mass index $\left(\mathrm{kg} / \mathrm{m}^{2}\right)$ & $23.6 \pm 3.35$ & $23.5 \pm 3.34$ & $24.0 \pm 3.36$ & $<0.001$ \\
\hline Diabetes & $25,214(5.3)$ & $19,934(5.1)$ & $5,280(6.1)$ & $<0.001$ \\
\hline Hypertension & $158,297(33.4)$ & $123,809(31.9)$ & $34,488(40.1)$ & $<0.001$ \\
\hline Current regular smoker & $126,194(26.6)$ & $102,991(26.6)$ & $23,203(27.0)$ & 0.010 \\
\hline Current regular drinker & $71,657(15.1)$ & $57,758(14.9)$ & $13,899(16.2)$ & $<0.001$ \\
\hline Physical activity (MET h/day) & $21.7 \pm 13.9$ & $21.8 \pm 13.9$ & $21.0 \pm 13.9$ & $<0.001$ \\
\hline
\end{tabular}

Values are presented as mean \pm standard deviation or $\mathrm{n}(\%)$.

MET, metabolic equivalent of task.

${ }^{*} P$-value refers to comparison between subjects with and without family history of stroke. For each quantitative variable, the $P$-value is obtained by the method of Student's t-test; for each categorical variable, the $P$-value is obtained through Pearson's $\chi^{2}$ test.

Table 2. HRs (95\% Cls) of incident stroke according to types of stroke and family history status

\begin{tabular}{|c|c|c|c|c|c|c|c|c|c|}
\hline \multirow{2}{*}{ Group } & \multirow{2}{*}{$\begin{array}{c}\text { Family history } \\
\text { status }\end{array}$} & \multirow{2}{*}{$\begin{array}{c}\text { No. of } \\
\text { subjects }\end{array}$} & \multirow{2}{*}{$\begin{array}{c}\text { Incident } \\
\text { strokes }\end{array}$} & \multirow{2}{*}{ Person-years } & \multirow{2}{*}{$\begin{array}{l}\text { Incidence rate } \\
\text { (per } 1,000 \\
\text { person years) }\end{array}$} & \multicolumn{2}{|c|}{ Model $1^{*}$} & \multicolumn{2}{|c|}{ Model $2^{+}$} \\
\hline & & & & & & $\mathrm{HR}(95 \% \mathrm{Cl})$ & $P_{\text {trend }}{ }^{\S}$ & $\mathrm{HR}(95 \% \mathrm{Cl})$ & $P_{\text {trend }}{ }^{\S}$ \\
\hline \multicolumn{10}{|c|}{ Total stroke ${ }^{\ddagger}$} \\
\hline & No & 387,864 & 19,988 & $2,734,939$ & 7.31 & 1.00 & & 1.00 & \\
\hline & Yes & 85,985 & 6,407 & 603,322 & 10.62 & $1.50(1.46-1.55)$ & & $1.34(1.31-1.38)$ & \\
\hline & 1 member & 74,931 & 5,158 & 527,282 & 9.78 & $1.41(1.37-1.46)$ & & $1.28(1.24-1.32)$ & \\
\hline & 2 members & 9,796 & 1,044 & 67,602 & 15.44 & $1.98(1.86-2.11)$ & & $1.66(1.56-1.77)$ & \\
\hline & $\geq 3$ members & 1,258 & 205 & 8,439 & 24.29 & $2.47(2.15-2.84)$ & $<0.001$ & $1.93(1.68-2.21)$ & $<0.001$ \\
\hline \multicolumn{10}{|c|}{ Hemorrhagic stroke } \\
\hline & No & 371,787 & 3,911 & $2,663,576$ & 1.47 & 1.00 & & 1.00 & \\
\hline & Yes & 80,635 & 1,057 & 579,429 & 1.82 & $1.28(1.20-1.37)$ & & 1.24 (1.16-1.33) & \\
\hline & 1 member & 70,634 & 861 & 508,128 & 1.69 & $1.21(1.13-1.31)$ & & $1.18(1.10-1.28)$ & \\
\hline & 2 members & 8,916 & 164 & 63,617 & 2.58 & $1.64(1.41-1.92)$ & & $1.53(1.31-1.79)$ & \\
\hline & $\geq 3$ members & 1,085 & 32 & 7,683 & 4.17 & $2.11(1.49-2.99)$ & $<0.001$ & 1.89 (1.33-2.67) & $<0.001$ \\
\hline \multicolumn{10}{|c|}{ Ischemic stroke } \\
\hline & No & 383,225 & 15,349 & $2,716,424$ & 5.65 & 1.00 & & 1.00 & \\
\hline & Yes & 84,757 & 5,179 & 598,374 & 8.66 & 1.59 (1.54-1.64) & & 1.39 (1.34-1.43) & \\
\hline & 1 member & 73,930 & 4,157 & 523,235 & 7.94 & $1.48(1.43-1.54)$ & & $1.32(1.27-1.36)$ & \\
\hline & 2 members & 9,604 & 852 & 66,854 & 12.74 & $2.11(1.97-2.26)$ & & $1.72(1.60-1.84)$ & \\
\hline & $\geq 3$ members & 1,223 & 170 & 8,285 & 20.52 & $2.69(2.31-3.13)$ & $<0.001$ & $2.01(1.73-2.34)$ & $<0.001$ \\
\hline
\end{tabular}

$\mathrm{HR}$, hazard ratio; $\mathrm{Cl}$, confidence interval.

*Model 1: adjusted for age and sex.

${ }^{\dagger}$ Model 2: Model 1 plus adjustment for marital status, education, residential area, smoking status, alcohol consumption, physical activity, body mass index, history of hypertension, history of diabetes and siblings.

${ }^{*}$ Including hemorrhagic stroke, ischemic stroke and stroke of unknown type.

${ }^{\S} P_{\text {trend }}$ : Linear trend test $P$-value for HRs' across number of members having stroke history for each stroke type within each model. 


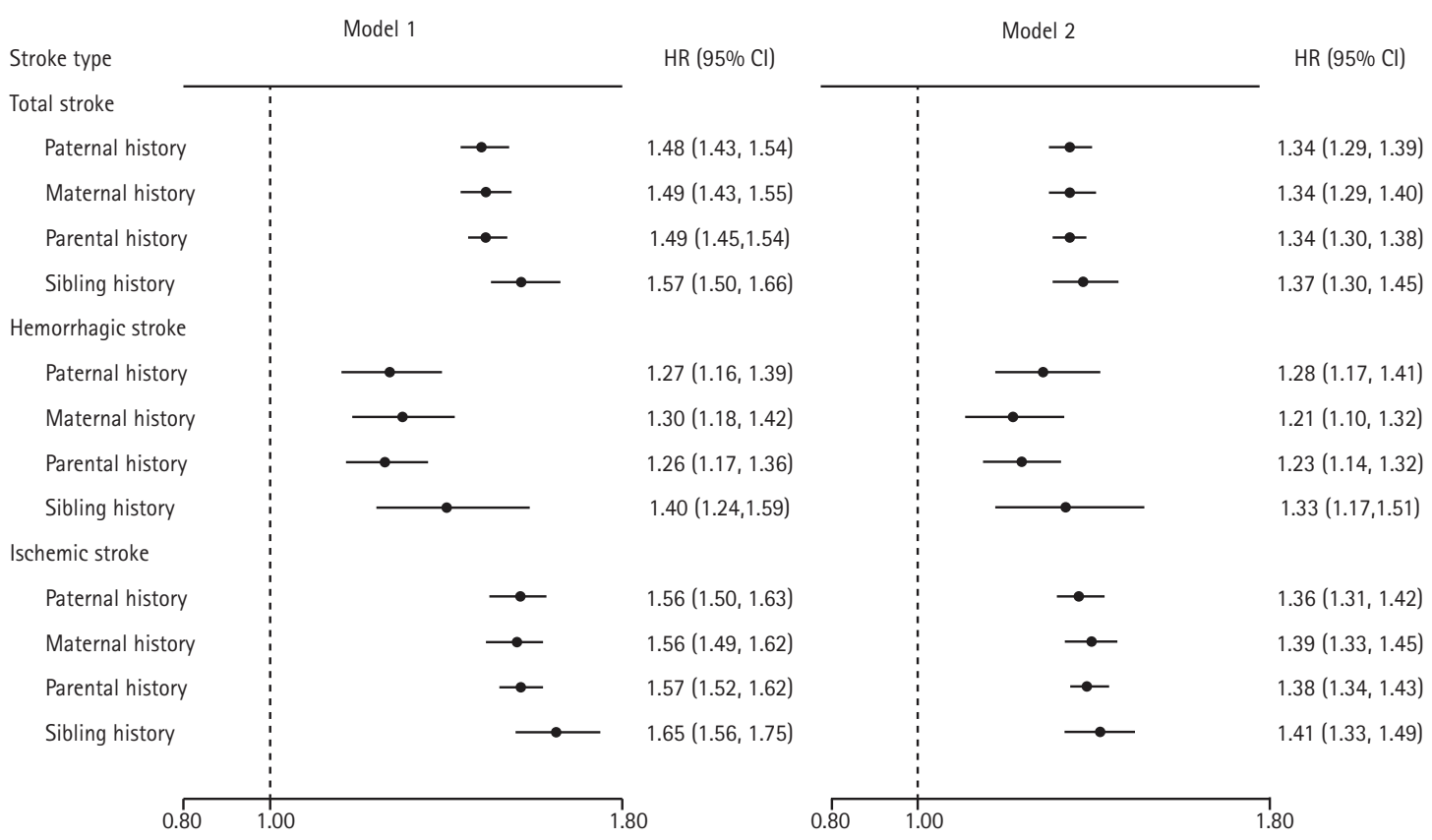

Figure 1. Hazard ratios (HRs) (95\% confidence intervals [Cls]) of incident stroke associated with each type of family history of stroke according to stroke types. Total stroke includes hemorrhagic stroke, ischemic stroke and stroke of unknown type. HRs and 95\% Cls of Model 1 are adjusted for age and sex; HRs and $95 \% \mathrm{Cls}$ of Model 2 are adjusted for age, sex, marital status, education, residential area, smoking status, alcohol consumption, physical activity, body mass index, history of hypertension, history of diabetes and siblings. Round dots represent the HRs, and horizontal lines represent the corresponding $95 \%$ Cls.
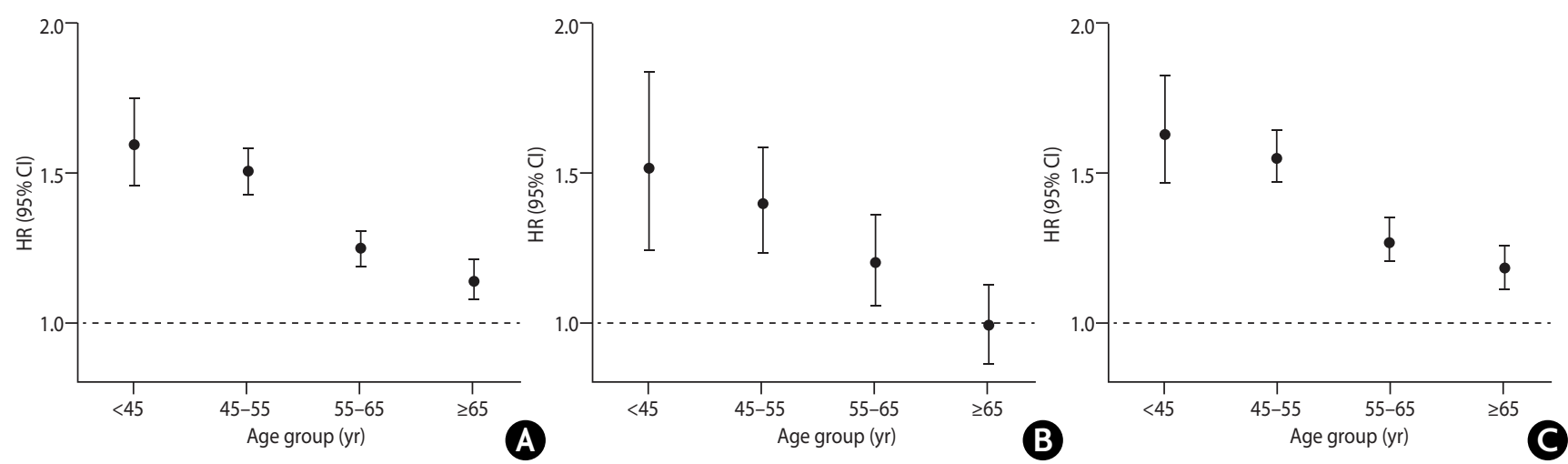

Figure 2. Hazard ratios (HRs) (95\% confidence intervals [Cls]) of incident stroke associated with family history of stroke according to age groups. (A) Results for total stroke; (B) results for hemorrhagic stroke; (C) results for ischemic stroke. Total stroke includes hemorrhagic stroke, ischemic stroke and stroke of unknown type. HRs and 95\% Cls are adjusted for sex, marital status, education, residential area, smoking status, alcohol consumption, physical activity, body mass index, history of hypertension, history of diabetes and siblings. Round dots represent the HRs, and horizontal lines represent the corresponding $95 \%$ Cls.

members with stroke, the age- and gender- adjusted HRs $(95 \%$ Cls) of stroke were 1.41 (1.37-1.46), 1.98 (1.86-2.11), and 2.47 (2.15-2.84) for subjects whose FHS involving 1,2 , and $\geq 3$ members $\left(P_{\text {trend }}<0.001\right)$, respectively, in comparison with those without FHS. The HRs increased with the increasing number of first degree relatives with stroke and this trend remained after multivariable adjustment ( $P_{\text {trend }}<0.001$ ). In Table 2 , the HRs for hemorrhagic stroke were increased with the number of first degree relatives with stroke, which also was the case for isch- emic stroke.

The age- and gender- adjusted HRs were 1.57 (95\% Cl: 1.501.66) and 1.49 (95\% $\mathrm{Cl}: 1.45-1.54)$ for sibling history and parental history, respectively, and the difference was not statistically significant $\left(P_{\text {neterogeneity }}=0.072\right)$ (Figure 1$)$. After the multivariable adjustment, the HR (95\% Cl) was 1.37 (1.30-1.45) for sibling history and 1.34 (1.30-1.38) for parental history ( $\left.P_{\text {neterogeneity }}=0.434\right)$. Age- and gender- adjusted HRs (95\% Cls) related to incident stroke were similar between individuals with paternal history 
(1.48 [1.43-1.54]) and maternal history (1.49 [1.43-1.55]) $\left(P_{\text {het- }}\right.$ erogeneity $=0.866)$. After multivariable adjustment, the HRs and 95\% Cls of incident stroke relevant to paternal history (1.34 [1.29-1.39]) and maternal history (1.34 [1.29-1.40]) remained comparable $\left(P_{\text {heterogeneity }}=0.846\right)$. Moreover, men and women had similar risk of incident stroke whenever their father or mother had stroke (all $P_{\text {heterogeneity }}>0.05$ between men and women in the associations of each type of FHS) (Supplementary Table 1). Similar patterns were also shown for both hemorrhagic and ischemic stroke in terms of the difference between sibling and parental history and in terms of the consistence between paternal and maternal history (Figure 1, Supplementary Table 1).

We further performed stratified analyses according to age groups (classified as $<45,45-54,55-64$, and $\geq 65 \mathrm{yr}$ ). We found that the HRs of incident stroke related to FHS decreased with age, and the HRs (95\% Cls) were 1.60 (1.46-1.76), 1.51 (1.431.59), 1.25 (1.19-1.31), and 1.14 (1.08-1.21), respectively (Figure 2). This trend was also obvious in both hemorrhagic and ischemic stroke in Figure 2. In the stratification analyses based on the other factors, individuals with FHS were more likely to be affected by stroke if they were currently tobacco smoking or alcohol drinking $(P<0.05$ after Bonferroni correction for heterogeneity test between subgroups) (Supplementary Table 2). Further analyses revealed significant interactions between these two health risk behaviors and FHS on the risk of stroke (Supplementary Table 2). Particularly, we observed a synergistic effect of FHS and current regular smoking on the risk of stroke regardless of types of stroke (Supplementary Figure 1).

\section{Discussion}

This Chinese cohort study showed that FHS was an independent risk factor for developing stroke, for whatever type of FHS. The more first degree relatives are affected by stroke, the higher are individuals' risk of suffering from stroke. Maternal history of stroke and paternal history of stroke had no differential effects on the risk of stroke, in both men and women. Individuals reported a positive FHS at young age had higher risk of stoke than those at late age. Moreover, FHS interacted with the health risk behaviors (smoking status and alcohol consumption) on the risk of stroke occurrence.

FHS represents a combination of genetic factors and shared environmental factors. It has been reported that compared with parent-offspring pairs, shared environmental effects on cardiovascular risk factors were stronger within sibling pairs. ${ }^{22,23}$ In addition, some studies reported that sibling history was more strongly correlated with the development and severity of stroke than parental history. ${ }^{14,24}$ Although the difference was not statistically significant, we found that sibling history was more strongly associated with stroke as opposed to parental history according to the purely numerical values. Additionally, this difference of purely numerical values between sibling history and parental history decreased after further adjusting for other covariates, including education, residential area, smoking and drinking status, which might highlight the contribution of shared environment to the sibling history of stroke.

Previous studies provided evidence for that the relationship between maternal history and stroke was different from that between paternal history and stroke. ${ }^{7,16-18}$ Touzé and Rothwell showed that the relationship between maternal and offspring stroke was stronger in females relative to males (sample size: 806), ${ }^{16}$ and this result was validated by a meta-analysis (total sample size: 7,941). ${ }^{19}$ However, Seshadri et al. reported that there was a marginally stronger association of paternal stroke with offspring stroke in comparison with maternal stroke (sample size: 3,443 ). ${ }^{7}$ Different from the above mentioned studies in Caucasian population, we firstly compared the differences of heritability of stroke between paternal and maternal history in this Chinese cohort study of 0.5 million individuals, and observed the same HRs for these two types of family history for both males and females. Thus, maternal history and paternal history need our equal attention in assessing heritability of stroke in Chinese population regardless of gender.

Numerous factors have been identified to be implicated with stroke occurrence in epidemiological research. ${ }^{25}$ In agreement with other studies, ${ }^{26,27}$ our study revealed that young age was more strongly associated with FHS in comparison with old age, which was consistent in both hemorrhagic stroke and ischemic stroke. A probable interpretation is that the relative importance of FHS and genetic factors may decrease with advancing age and other postnatal factors may become more prominent. ${ }^{28}$ In addition, we found significant interactions between FHS and two health-risk behaviors, including smoking and alcohol consumption. Particularly, we identified a synergistic effect of FHS and smoking on the risk of stroke regardless of types of stroke. Smoking was one of the major risk factors of stroke and might affect the concentration of high density lipoprotein, increase blood fibrinogen levels, promote atherosclerosis formation, and further increase the risk of hypertension and diabetes (modifiable risk factors of stroke). ${ }^{29,30}$ Thus, smoking would increase the incidence risk of stroke in Chinese population, especially for the individuals with FHS, which emphasizing the importance of management of smoking. Similarly, alcohol drinking is also related to stroke risk, especially for the individuals with FHS. Therefore, the importance of management of the health risk behaviors for reducing stroke risk should be highlighted for 
Chinese population, especially for the individuals with FHS.

Strengths of our study are the prospective cohort design, the large sample size, the well-designed questionnaire, and the efficient data collection and management. There are also some limitations that need to be mentioned. First, we did not obtain the information about the age of onset of parental stroke. Thus, we could not explore the association of early-onset stroke in parents to incident stroke in offspring. Second, family history data were obtained based on self-report, which might affect the accuracy of the information and lead to some degree of misclassification bias. However, in self-reported surveys, the recall bias is inevitable, and in comparison with case-control studies, the recall bias was less in our cohort study. Besides, previous studies have reported that self-reported history is reliable for stroke data. ${ }^{31}$ Third, some stroke risk factors were not adjusted for (such as serum total cholesterol) because the relevant information was not collected. These factors may partly explain the stroke risk associated with FHS.

\section{Conclusions}

FHS is an independent risk factor for stroke in Chinese, and the more first degree relatives are affected by stroke, the higher are individuals' risk of suffering from stroke. The management of the health risk behaviors for reducing stroke should be highlighted, especially for the individuals with FHS.

\section{Acknowledgments}

The chief acknowledgment is to the participants, the project staff, and the China National Centre for Disease Control and Prevention $(C D C)$ and its regional offices for assisting with the fieldwork. We thank Judith Mackay in Hong Kong; Yu Wang, Gonghuan Yang, Zhengfu Qiang, Lin Feng, Maigeng Zhou, Wenhua Zhao, and Yan Zhang in China CDC; Lingzhi Kong, Xiucheng $Y u$, and Kun Li in the Chinese Ministry of Health; and Sarah Clark, Martin Radley, Mike Hill, Hongchao Pan, and Jill Boreham in the CTSU, Oxford, for assisting with the design, planning, organization, and conduct of the study.

Details for the CKB collaborative group are given in the Appendix of Online supplement.

\section{Supplementary Materials}

Supplementary materials related to this article can be found online at https://doi.org/10.5853/jos.2016.01270.

\section{References}

1. GBD 2013 Mortality and Causes of Death Collaborators. Global, regional, and national age-sex specific all-cause and cause-specific mortality for 240 causes of death, 19902013: a systematic analysis for the Global Burden of Disease Study 2013. Lancet 2015;385:117-171.

2. Liu L, Wang D, Wong KS, Wang Y. Stroke and stroke care in China: huge burden, significant workload, and a national priority. Stroke 2011;42:3651-3654.

3. Pearson TA, Blair SN, Daniels SR, Eckel RH, Fair JM, Fortmann $\mathrm{SP}$, et al. AHA guidelines for primary prevention of cardiovascular disease and stroke: 2002 update: consensus panel guide to comprehensive risk reduction for adult patients without coronary or other atherosclerotic vascular diseases. Circulation 2002;106:388-391.

4. Eguchi E, Iso H, Wada Y, Kikuchi S, Watanabe $Y$, Tamakoshi $A_{i}$ Japan Collaborative Cohort Study Group. Parental history and lifestyle behaviors in relation to mortality from stroke among Japanese men and women: the Japan Collaborative Cohort Study. J Epidemiol 2012;22:331-339.

5. Kadota A, Okamura T, Hozawa A, Kadowaki T, Murakami $Y_{\text {, }}$ Hayakawa $T$, et al. Relationships between family histories of stroke and of hypertension and stroke mortality: NIPPON DATA80, 1980-1999. Hypertens Res 2008;31:1525-1531.

6. Khaw KT, Barrett-Connor E. Family history of stroke as an independent predictor of ischemic heart disease in men and stroke in women. Am J Epidemiol 1986;123:59-66.

7. Seshadri S, Beiser A, Pikula A, Himali JJ, Kelly-Hayes M, Debette $S$, et al. Parental occurrence of stroke and risk of stroke in their children: the Framingham study. Circulation 2010;121:1304-1312.

8. Kiely DK, Wolf PA, Cupples LA, Beiser AS, Myers RH. Familial aggregation of stroke. The Framingham Study. Stroke 1993;24:1366-1371.

9. Jousilahti $P$, Rastenyte $D$, Tuomilehto J, Sarti C, Vartiainen E. Parental history of cardiovascular disease and risk of stroke. A prospective follow-up of 14371 middle-aged men and women in Finland. Stroke 1997;28:1361-1366.

10. Morrison AC, Fornage $M$, Liao D, Boerwinkle E. Parental history of stroke predicts subclinical but not clinical stroke: the Atherosclerosis Risk in Communities Study. Stroke 2000;31:2098-2102.

11. Wannamethee SG, Shaper AG, Ebrahim S. History of parental death from stroke or heart trouble and the risk of stroke in middle-aged men. Stroke 1996;27:1492-1498.

12. Yanez ND, Burke GL, Manolio T, Gardin JM, Polak J; CHS Collaborative Research Group. Sibling history of myocardial in- 
farction or stroke and risk of cardiovascular disease in the elderly: the Cardiovascular Health Study. Ann Epidemiol 2009;19:858-866.

13. Zhang LF, Wu YF, Li Y, Li X, Xie GQ, Zhao LC. A cohort study on parental history and risk factors of stroke in Chinese population. Zhonghua Liu Xing Bing Xue Za Zhi 2007;28:10601063.

14. Choi JC, Lee JS, Kang SY, Kang JH, Bae JM. Family history and risk for ischemic stroke: sibling history is more strongly correlated with the disease than parental history. J Neurol Sci 2009;284:29-32.

15. Polychronopoulos P, Gioldasis G, Ellul J, Metallinos IC, Lekka $N P$, Paschalis $C$, et al. Family history of stroke in stroke types and subtypes. J Neurol Sci 2002;195:117-122.

16. Touzé $E_{1}$ Rothwell PM. Heritability of ischaemic stroke in women compared with men: a genetic epidemiological study. Lancet Neuro/ 2007;6:125-133.

17. Liao D, Myers R, Hunt $S$, Shahar E, Paton C, Burke G, et al. Familial history of stroke and stroke risk. The Family Heart Study. Stroke 1997;28:1908-1912.

18. Caicoya $M$, Corrales $C$, Rodriguez T. Family history and stroke: a community case-control study in Asturias, Spain. J Epidemiol Biostat 1999;4:313-320.

19. Touzé $E_{1}$ Rothwell PM. Sex differences in heritability of ischemic stroke: a systematic review and meta-analysis. Stroke 2008;39:16-23.

20. Chen Z, Lee L, Chen J, Collins R, Wu F, Guo Y, et al. Cohort profile: the Kadoorie Study of Chronic Disease in China (KSCDC). Int J Epidemiol 2005;34:1243-1249.

21. Chen $Z$, Chen J, Collins $R$, Guo $Y$, Peto $R$, Wu F, et al. China Kadoorie Biobank of 0.5 million people: survey methods, baseline characteristics and long-term follow-up. Int J Epidemiol 2011;40:1652-1666.

22. Knuiman MW, Divitini ML, Welborn TA, Bartholomew HC. Familial correlations, cohabitation effects, and heritability for cardiovascular risk factors. Ann Epidemiol 1996;6:188194.
23. Harrap SB, Stebbing M, Hopper JL, Hoang HN, Giles GG. Familial patterns of covariation for cardiovascular risk factors in adults: the Victorian Family Heart Study. Am J Epidemiol 2000;152:704-715.

24. Meschia JF, Case LD, Worrall BB, Brown RD Jr, Brott TG, Frankel $M$, et al. Family history of stroke and severity of neurologic deficit after stroke. Neurology 2006;67:1396-1402.

25. Huang HD, Yang CM, Shu HF, Kuang YQ, Yang T, He WQ, et al. Genetic predisposition of stroke: understanding the evolving landscape through meta-analysis. Int J Clin Exp Med 2015;8:1315-1323.

26. Hassan A, Sham PC, Markus HS. Planning genetic studies in human stroke: sample size estimates based on family history data. Neurology 2002;58:1483-1488.

27. Williams RR, Hunt SC, Heiss G, Province MA, Bensen JT, Higgins $M$, et al. Usefulness of cardiovascular family history data for population-based preventive medicine and medical research (the Health Family Tree Study and the NHLBI Family Heart Study). Am J Cardiol 2001;87:129-135.

28. Kulshreshtha A, Vaccarino $V$, Goyal $A$, McClellan W, Nahab $F$, Howard VJ, et al. Family history of stroke and cardiovascular health in a national cohort. J Stroke Cerebrovasc Dis 2015;24:447-454.

29. Freeman DJ, Griffin BA, Murray E, Lindsay GM, Gaffney $D$, Packard CJ, et al. Smoking and plasma lipoproteins in man: effects on low density lipoprotein cholesterol levels and high density lipoprotein subfraction distribution. Eur J Clin Invest 1993;23:630-640.

30. Lekakis J, Papamichael C, Vemmos C, Stamatelopoulos K, Voutsas A, Stamatelopoulos, S. Effects of acute cigarette smoking on endothelium-dependent arterial dilatation in normal subjects. Am J Cardiol 1998;81:1225-1228.

31. Okura Y, Urban LH, Mahoney DW, Jacobsen SJ, Rodeheffer RJ. Agreement between self-report questionnaires and medical record data was substantial for diabetes, hypertension, myocardial infarction and stroke but not for heart failure. $J$ Clin Epidemiol 2004;57:1096-1103. 
Supplementary Table 1. Multivariable adjusted hazard ratios (95\% confidence intervals) of incident stroke associated with family history of stroke according to stroke types and gender

\begin{tabular}{|c|c|c|c|c|c|c|}
\hline \multirow{2}{*}{ Outcome } & \multirow{2}{*}{ Gender } & Family history & Paternal history & Maternal history & Parental history & Sibling history \\
\hline & & $\operatorname{HR}(95 \% \mathrm{CI})^{*}$ & $\operatorname{HR}(95 \% \mathrm{CI})^{*}$ & $\operatorname{HR}(95 \% \mathrm{Cl})^{*}$ & $\operatorname{HR}(95 \% \mathrm{Cl})^{*}$ & $\operatorname{HR}(95 \% \mathrm{CI})^{*}$ \\
\hline \multirow[t]{2}{*}{ Total stroke $^{+}$} & Male & $1.35(1.29-1.41)$ & $1.34(1.27-1.42)$ & $1.33(1.26-1.41)$ & $1.35(1.29-1.41)$ & $1.35(1.25-1.45)$ \\
\hline & Female & $1.33(1.28-1.38)$ & $1.33(1.26-1.40)$ & $1.34(1.27-1.41)$ & $1.33(1.27-1.38)$ & $1.39(1.30-1.49)$ \\
\hline \multirow[t]{2}{*}{ Hemorrhagic stroke } & Male & $1.22(1.11-1.35)$ & $1.28(1.12-1.45)$ & $1.22(1.07-1.39)$ & $1.23(1.11-1.37)$ & $1.17(0.97-1.41)$ \\
\hline & Female & $1.26(1.14-1.39)$ & $1.29(1.13-1.48)$ & $1.19(1.05-1.36)$ & $1.22(1.10-1.35)$ & $1.50(1.26-1.77)$ \\
\hline \multirow[t]{2}{*}{ Ischemic stroke } & Male & $1.40(1.34-1.47)$ & $1.37(1.29-1.46)$ & $1.39(1.30-1.48)$ & $1.39(1.33-1.46)$ & $1.42(1.30-1.55)$ \\
\hline & Female & $1.36(1.30-1.42)$ & $1.35(1.27-1.43)$ & $1.38(1.30-1.46)$ & $1.36(1.30-1.43)$ & $1.39(1.29-1.51)$ \\
\hline
\end{tabular}

$\mathrm{HR}$, hazard ratio; $\mathrm{Cl}$, confidence interval.

${ }^{*}$ Adjusted for age, marital status, education, residential area, smoking status, alcohol consumption, physical activity, BMl, history of hypertension, history of diabetes and siblings.

'Including hemorrhagic stroke, ischemic stroke and stroke of unknown type. 
Supplementary Table 2. Stratification analyses on associations between family history of stroke and incident stroke

\begin{tabular}{|c|c|c|c|c|}
\hline Subgroup & $H R^{*}$ & $95 \% \mathrm{Cl}^{*}$ & $\begin{array}{c}P \\
\text { for heterogeneity }\end{array}$ & $\begin{array}{c}P \\
\text { for interaction }\end{array}$ \\
\hline Residential area & & & 0.145 & \\
\hline Rural & 1.37 & $1.32-1.43$ & & \\
\hline Urban & 1.31 & $1.26-1.37$ & & \\
\hline Marital status & & & 0.012 & \\
\hline Married & 1.36 & $1.32-1.40$ & & \\
\hline Widowed/separated/divorced/never married & 1.22 & $1.13-1.32$ & & \\
\hline Education & & & $<0.001$ & $<0.001$ \\
\hline Illiteracy or primary school & 1.26 & $1.21-1.31$ & & \\
\hline Middle school and above & 1.44 & $1.38-1.50$ & & \\
\hline Smoking status & & & $<0.001$ & $<0.001$ \\
\hline Not current & 1.30 & $1.26-1.34$ & & \\
\hline Current & 1.46 & $1.38-1.54$ & & \\
\hline Alcohol consumption & & & 0.005 & $<0.001$ \\
\hline Not current & 1.31 & $1.27-1.36$ & & \\
\hline Current & 1.45 & $1.36-1.53$ & & \\
\hline Physical activity (MET h/day) & & & $<0.001$ & $<0.001$ \\
\hline$<18.20$ & 1.29 & $1.25-1.34$ & & \\
\hline$\geq 18.20$ & 1.46 & $1.39-1.54$ & & \\
\hline BMI $\left(\mathrm{kg} / \mathrm{m}^{2}\right)$ & & & 0.788 & \\
\hline$<24.0$ & 1.34 & $1.28-1.40$ & & \\
\hline $24.0,27.9$ & 1.36 & $1.30-1.42$ & & \\
\hline$\geq 28.0$ & 1.32 & $1.23-1.42$ & & \\
\hline Hypertension & & & 0.035 & \\
\hline No & 1.39 & $1.32-1.46$ & & \\
\hline Yes & 1.30 & $1.26-1.35$ & & \\
\hline Diabetes & & & 0.034 & \\
\hline No & 1.36 & $1.32-1.40$ & & \\
\hline Yes & 1.24 & $1.14-1.34$ & & \\
\hline
\end{tabular}

$\mathrm{HR}$, hazard ratio; $\mathrm{Cl}$, confidence interval; MET, metabolic equivalent of task; $\mathrm{BMI}$, body mass index.

*Adjusted for age, sex, marital status, education, residential area, smoking status, alcohol consumption, physical activity, BMI, history of hypertension, history of diabetes and siblings except for the stratification factor. 


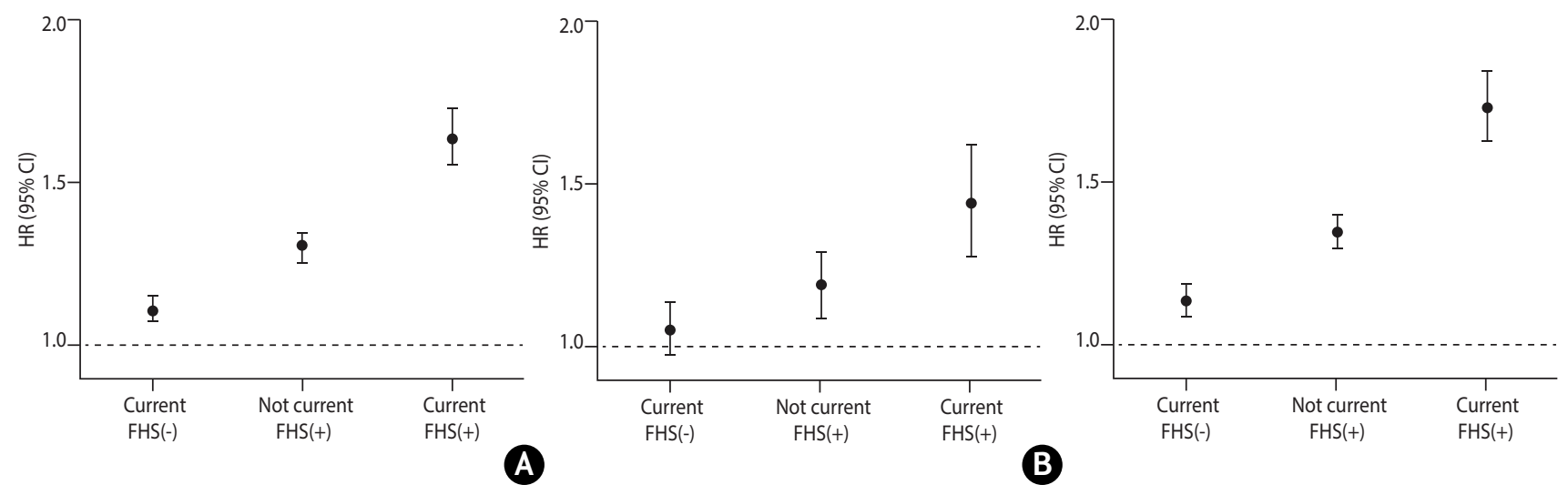

Supplementary Figure 1. Interaction analyses between family history of stroke and smoking status. (A) Results for total stroke; (B) results for hemorrhagic stroke; (C) results for ischemic stroke. Total stroke includes hemorrhagic stroke, ischemic stroke and stroke of unknown type. HRs and 95\% Cls are adjusted for age, sex, marital status, education, residential area, alcohol consumption, physical activity, BMI, history of hypertension, history of diabetes and siblings. Round dots represent the HRs, and horizontal lines represent the corresponding 95\% Cls. Current refers to current regular smoker. Not current refers to not current regular smoker. FHS(-) refers to without FHS. FHS(+) refers to with FHS. The dashed lines represent participants who are not current smokers and without FHS as reference groups. FHS, family history of stroke; HR, hazard ratio; $\mathrm{Cl}$, confidence interval; BMI, body mass index. 


\section{Appendix}

Details for members of the China Kadoorie Biobank collaborative group: International Steering Committee: Junshi Chen, Zhengming Chen (PI), Rory Collins, Liming Li (PI), Richard Peto. International Co-ordinating Centre, Oxford: Daniel Avery, Derrick Bennett, Yumei Chang, Yiping Chen, Zhengming Chen, Robert Clarke, Huaidong Du, Xuejuan Fan, Simon Gilbert, Alex Hacker, Michael Holmes, Andri lona, Christiana Kartsonaki, Rene Kerosi, Ling Kong, Om Kurmi, Garry Lancaster, Sarah Lewington, John McDonnell, Winnie Mei, lona Millwood, Qunhua Nie, Jayakrishnan Radhakrishnan, Sajjad Rafiq, Paul Ryder, Sam Sansome, Dan Schmidt, Paul Sherliker, Rajani Sohoni, lain Turnbull, Robin Walters, Jenny Wang, Lin Wang, Ling Yang, Xiaoming Yang. National Co-ordinating Centre, Beijing: Zheng Bian, Ge Chen, Yu Guo, Bingyang Han, Can Hou, Jun Lv, Pei Pei, Shuzhen Qu, Yunlong Tan, Canqing Yu, Huiyan Zhou. 10 Regional Co-ordinating Centres: Oingdao CDC: Zengchang Pang, Ruqin Gao, Shaojie Wang, Yongmei Liu, Ranran Du, Yajing Zang, Liang Cheng, Xiaocao Tian, Hua Zhang. Licang CDC: Silu Lv, Junzheng Wang, Wei Hou. Heilongjiang Provincial CDC: Jiyuan Yin, Ge Jiang, Shumei Liu, Zhigang Pang, Xue Zhou. Nangang CDC: Liqiu Yang, Hui He, Bo Yu, Yanjie Li, Huaiyi Mu, Qinai Xu, Meiling Dou, Jiaojiao Ren. Hainan Provincial CDC: Jianwei Du, Shanq- ing Wang, Ximin Hu, Hongmei Wang, Jinyan Chen, Yan Fu, Zhenwang Fu, Xiaohuan Wang, Hua Dong. Meilan CDC: Min Weng, Xiangyang Zheng, Yijun Li, Huimei Li, Chenglong Li. Jiangsu Provincial CDC: Ming Wu, Jinyi Zhou, Ran Tao, Jie Yang. Suzhou CDC: Jie Shen, Yihe Hu, Yan Lu, Yan Gao, Liangcai Ma, Renxian Zhou, Aiyu Tang, Shuo Zhang, Jianrong Jin. Guangxi Provincial CDC: Zhenzhu Tang, Naying Chen, Ying Huang. Liuzhou CDC: Mingqiang Li, Jinhuai Meng, Rong Pan, Qilian Jiang, Jingxin Oing, Weiyuan Zhang, Yun Liu, Liuping Wei, Liyuan Zhou, Ningyu Chen, Jun Yang, Hairong Guan. Sichuan Provincial CDC: Xianping Wu, Ningmei Zhang, Xiaofang Chen, Xuefeng Tang. Pengzhou CDC: Guojin Luo, Jianguo Li, Xiaofang Chen, Jian Wang, Jiaqiu Liu, Qiang Sun. Gansu Provincial CDC: Pengfei Ge, Xiaolan Ren, Caixia Dong. Maiji CDC: Hui Zhang, Enke Mao, Xiaoping Wang, Tao Wang. Henan Provincial CDC: Guohua Liu, Baoyu Zhu, Gang Zhou, Shixian Feng, Liang Chang, Lei Fan. Huixian CDC: Yulian Gao, Tianyou He, Li Jiang, Huarong Sun, Pan He, Chen Hu, Qiannan Lv, Xukui Zhang. Zhejiang Provincial CDC: Min Yu, Ruying Hu, Le Fang, Hao Wang. Tongxiang CDC: Yijian Qian, Chunmei Wang, Kaixue Xie, Lingli Chen, Yaxing Pan, Dongxia Pan. Hunan Provincial CDC: Yuelong Huang, Biyun Chen, Donghui Jin, Huilin Liu, Zhongxi Fu, Qiaohua Xu. Liuyang CDC: Xin Xu, Youping Xiong, Weifang Jia, Xianzhi Li, Libo Zhang, Zhe Qiu. 